\title{
Transmembrane segment M2 of glycine receptor as a model system for the pore-forming structure of ion channels ${ }^{\star}$
}

\author{
Piotr Bednarczyk ${ }^{1}$, Adam Szewczyk ${ }^{2}$ and Krzysztof Dołowy ${ }^{1 凶}$ \\ ${ }^{1}$ Department of Biophysics, Agricultural University SGGW, Warszawa, Poland; ${ }^{2}$ Laboratory \\ of Intracellular Ion Channels, Nencki Institute of Experimental Biology, Polish Academy of \\ Sciences, Warszawa, Poland
}

Received: 20 October, 2002; revised: 21 November, 2002; accepted: 04 December, 2002

Key words: glycine receptor, ion channels, black lipid membrane

\begin{abstract}
The glycine receptor belongs to the ligand-gated ion channel superfamily. It is a chloride conducting channel composed of four transmembrane domains. It was previously shown that the second transmembrane domain (M2) of the glycine receptor forms an ion conduction pathway throught lipid bilayers. The amino-acid sequence of the transmembrane segment M2 of the glycine receptor has a high homology to all receptors of the ligand-gated ion channel superfamily. In our report, we have used a synthetic M2 peptide. It was incorporated into a planar membrane of known lipid composition and currents induced by M2 were measured by the Black Lipid Membrane technique. When the planar lipid bilayer was composed of $75 \%$ phosphatidylethanolamine and $25 \%$ phosphatidylserine, the reversal potential measured in a 150/600 $\mathrm{mM} \mathrm{KCl}$ (cis/trans) gradient was $-19 \mathrm{mV}$ suggesting that the examined pore was preferential to anions, $\mathbf{P}_{\mathbf{K}} / \mathbf{P}_{\mathrm{Cl}}=0.25$. In contrast, when $75 \%$ phosphatidylserine and $25 \%$ phosphatidylethanolamine was used, the reversal potential was $+20 \mathrm{mV}$ and the pore was preferential to cations, $P_{K} / P_{C l}=4.36$. Single-channel currents were recorded with two predominant amplitudes corresponding to the main-conductance and sub-conductance states. Both conductance states (about $12 \mathrm{pS}$ and $30 \mathrm{pS}$ ) were measured in a symmetric solution of $50 \mathrm{mM} \mathrm{KCl}$. The observed single-channel properties suggest that the selectivity and conductance of the pore formed by the M2 peptide of the glycine receptor depend on the lipid composition of the planar bilayer.
\end{abstract}

\footnotetext{
This work was supported by a grant from the President of the SGGW No. 50406020013 and the Nencki Institute of Experimental Biology.

${ }^{\otimes}$ Corresponding author: Krzysztof Dołowy, Department of Biophysics, Agricultural University SGGW, Rakowiecka 26/30, 02-528 Warszawa, Poland; tel.: (48 22) 8492251 ext. 2285, fax: (48 22) 849 1676, e-mail: dolowy@delta.sggw.waw.pl

Abbreviations: BLM, black lipid membrane; $\mathrm{GABA}_{\mathrm{A}} \mathrm{R}$ and $\mathrm{GABA}_{\mathrm{C}} \mathrm{R}, \boldsymbol{\gamma}$-aminobutyric acid $\mathrm{A}$ and $\mathrm{C}$ subtype receptors; GlyR, glycine receptor; 5 - $\mathrm{HT}_{3} \mathrm{R}$, 5-hydroxytryptamine type 3 receptor; LGICS, ligand-gated ion channel superfamily; M2, transmembrane segment of glycine receptor; nAChR, nicotinic acetylcholine receptor; $\mathrm{PE}$, phosphatidylethanolamine (1-palmitoyl-2-oleoyl-sn-glycero-3-phosphoethanolamine); PS, phosphatidylserine (1-palmitoyl-2-oleoyl-sn-glycero-3-[phospho-L-serine]); TFE, 2,2,2-trifluoroethanol.
} 
Nicotinic acetylcholine receptor (nAChR), $\gamma$-aminobutric acid $A$ and $C$ subtype receptors $\left(\mathrm{GABA}_{\mathrm{A}} \mathrm{R}\right.$ and $\left.\mathrm{GABA}_{\mathrm{C}} \mathrm{R}\right)$, glycine receptor (GlyR) and 5-hydroxytryptamine type 3 receptor $\left(5-\mathrm{HT}_{3} \mathrm{R}\right)$ are members of the ligand-gated ion channel superfamily (LGICS). Members of this ion channel superfamily share certain structural and functional properties (Leite \& Cascio, 2001; Adcock et al., 1998). Each channel molecule is composed of five subunits. Each subunit has four hydrophobic segments M1-M4. M2 forms a narrow region of the ion conduction pathway (Fig. 1A).

The best-characterized member of this family is the nicotinic acetylcholine receptor, which mediates neurotransmission at vertebrate neuromuscular junctions. It also plays a role at some synapses of the vertebrate central nervous system and in the nervous system of invertebrates (e.g. Nicholls et al., 2001). Both functional and structural studies on $\mathrm{nAChR}$ indicate that it undergoes a conformational change upon binding two neurotransmitter (acetylcholine) molecules, switching from a closed to an open state. The open state allows passage of monovalent and divalent cations by way of a pore that runs through the center of the protein. Other LGICS, such as GlyR, are permeable to anions (Kuhse et al., 1995). The anion-selective LGICS mediate inhibitory neurotransmission, whereas the cation selective ones are excitatory. Other members of the superfamily include the anion-selective $\mathrm{GABA}_{\mathrm{A}} \mathrm{R}$ (Sieghart \& Sperk, 2002; Sieghart, 1995), GABA $_{C} R$ (Bormann \& Feigenspan, 1995) and the cation-selective $5 \mathrm{HT}_{3}$ receptor (Reeves \& Lummis, 2002).

The single-channel properties of the glycine receptor have been investigated in detail by patch clamp recordings from embryonic mouse spinal cord neurons in culture (Twyman \& Macdonald, 1991). The channel is selective for monovalent anions and exhibits multiple conductance states (Keramidas et al., 2002). A synthetic peptide, with a sequence corresponding to the M2 segment of GlyR, forms an anion selective pore in lipid bilayers (Reddy et al., 1993; Langosch et al., 1991). The properties of M2 peptide modified by adding four lysine residues to the $\mathrm{C}$-terminus are similar to those described for the parent sequence (Wallace et al., 2000). Single-channel currents induced by the M2 peptide show two predominant amplitudes corresponding to a main conductance states of about $25 \mathrm{pS}$ and $49 \mathrm{pS}$ in symmetric $500 \mathrm{mM} \mathrm{KCl}$ with channel open lifetimes in the milliseconds time range (Reddy et al., 1993).

The phospholipid composition of the lipid bilayer plays an important role in membrane transport (Sprong et al., 2001). The charge of the lipid polar groups and ion absorption create electrostatic potential that modulates the concentration of protons, ions, and other charged substrates in the vicinity of the membrane (Nalecz et al., 1980). Understanding these regulatory processes requires knowledge of the lipid polar group structure, organization, and the state of ionization. The membrane surface charge modifies the conductance of multi-state channels formed in planar lipid bilayers by the peptide antibiotic alamethicin (Aguilella \& Bezrukov, 2001) and gramicidin A (de Godoy \& Cukierman, 2001; Rostovtseva et al., 1998). Fan and Makielski (1997) show that anionic phospholipids activate ATP-sensitive potassium channels. It has also been shown that the conductance of the $\mathrm{K}^{+}$channel of the sarcoplasmic reticulum increases with an increase in the number of negative charges in the membrane (Anzai et al., 1994).

In our report, the channel activity of a synthetic M2 peptide from the glycine receptor was measured after reconstitution into lipid bilayers. We observed single-channel currents with two predominant amplitudes corresponding to the main-conductance and sub-conductance states. When the planar lipid bilayer was composed of uncharged phospholipids the reversal potential measurements suggested that the examined pore was preferential to anions. In contrast, when negative 
phospholipids were used for bilayer formation, the pore induced by the M2 peptide was preferential to cations.

\section{MATERIALS AND METHODS}

Materials. 1-Palmitoyl-2-oleoyl-sn-glycero-3phosphoethanolamine (PE) and 1-palmitoyl2-oleoyl-sn-glycero-3-[phospho-L-serine] (PS) were from Avanti Polar Lipids Inc. (U.S.A.). 2,2,2-Trifluoroethanol (TFE) and n-decane were from Sigma-Aldrich, Germany. All other chemicals were of the highest purity available commercially.

Peptide synthesis. The M2 peptide was synthesized by the solid-phase method using Fmoc chemistry. The crude peptide was purified on a semipreparative C8 HPLC column. The purity of the peptide was greater than $98 \%$. The synthesized peptide gave the correct molecular mass as measured by mass spectroscopy using the MALDI-TOF technique. The amino-acid sequence of the synthetic M2 peptide used in this study is shown in Fig. 1B. The M2 peptide corresponds to the M2 segment (amino acids 250-272) of the $\alpha$ subunit of the strychnine-sensitive subunit of the inhibitory glycine receptor from human brain.

Black lipid membrane measurements. Planar phospholipid bilayers were formed in a $250 \mu \mathrm{m}$ diameter hole drilled in a Delrin cup (Warner Instrument Corp., Hamden, CT. U.S.A.), which separated two chambers (cis 1 $\mathrm{ml}$ and trans $1 \mathrm{ml}$ internal volume). The chambers contained 150/600 mM KCl (cis/trans), $20 \mathrm{mM}$ Hepes, $\mathrm{pH}$ 7.0. The outline of the aperture was coated with a lipid solution and $\mathrm{N}_{2}$ dried prior to bilayer formation to improve membrane stability. Planar phospholipid bilayers were painted using $3: 1$ or $1: 3$ mixed solutions of the synthetic lipids PE:PS in $\mathrm{n}$-decane at a final concentration of $25 \mathrm{mg}$ $\mathrm{lipid} / \mathrm{ml}$. Stock solutions ( $1 \mathrm{mg} / \mathrm{ml}$ ) of M2 peptides in TFE were added to the trans compartment. The M2 peptide was incorporated into the black lipid membrane within a few min- utes. All measurements were carried out at room temperature. Formation and thinning of the bilayer was monitored by capacitance measurements and optical observations. The final accepted capacitance values ranged from 110 to $180 \mathrm{pF}$. Electrical connections were made by $\mathrm{Ag} / \mathrm{AgCl}$ electrodes and agar salt bridges $(3 \mathrm{M} \mathrm{KCl})$ to minimize liquid junction potentials. The current was measured using a Bilayer Membrane Amplifier (BLM-120, BioLogic).

Data analysis. Signals were filtered at 200 $\mathrm{Hz}$, digitized (A/D converter PowerLab 2/20, ADInstruments) and transferred to a $\mathrm{PC}$ or digital tape recorder (DTR-1204, BioLogic) for off-line analysis by Chart v4.1.2 (PowerLab, ADInstruments) and pCLAMP8 (Axon Instruments). Conductance values were calculated from current histograms best fitted by a sum of two Gaussian distributions. The permeability ratios for $\mathrm{K}^{+}$and $\mathrm{Cl}^{-}$were calculated according to the Goldman-Hodgkin-Katz voltage equation (Hille, 2001):

$$
E_{\text {rev }}=-\frac{R T}{z F} \ln \frac{[C l]_{\text {cis }}+{ }^{P_{K}} /_{P_{C l}}[K]_{\text {trans }}}{[C l]_{\text {trans }}+{ }^{P_{K}} /_{P_{C l}}[K]_{c i s}},
$$

where $E_{\text {rev }}$ is the potential at which the current is zero, $R$ is the gas constant, $T$ is temperature in Kelvin, $z=-1$ (chloride anion charge), $F$ is Faraday's constant, $P_{\text {ion }}$ is the permeability of the ion, and [ion] is the concentration of the ion in the cis and trans chambers, respectively. We can rewrite Eqn. 1 as

$$
P_{K} /_{P_{C l}}=\frac{\xi[C l]_{t r a n s}-[C l]_{c i s}}{[K]_{\text {trans }}-\xi[C l]_{c i s}},
$$

where $\xi=\exp \left(-\frac{z F E_{r e v}}{R T}\right)$

\section{RESULTS AND DISCUSSION}

The glycine receptor is a chloride conducting channel (Twyman \& Macdonald, 1991). The 
channel molecule is composed of five subunits. Each subunit has four hydrophobic segments M1-M4. The M2 segment forms an ion conduction pathway through lipid bilayers (Reddy et al., 1993; Langosch et al., 1991). It is known that the amino-acid sequence of M2 influences the properties of the pore. Reddy et al. (1993) postulated that two arginine residues in the M2 peptide (Fig. 1B) determine served. Current-time traces were recorded in the $150 / 600 \mathrm{mM} \mathrm{KCl}$ gradient (cis/trans) at different voltages (Fig. 2A) with the planar lipid bilayers composed of PE:PS=3:1 phospholipids (number of observations, $n=5$ ). Fig. 2B shows current-voltage curves for single channel openings at different voltages under the same conditions. Solid and dashed lines indicate the main-conductance and sub-con-

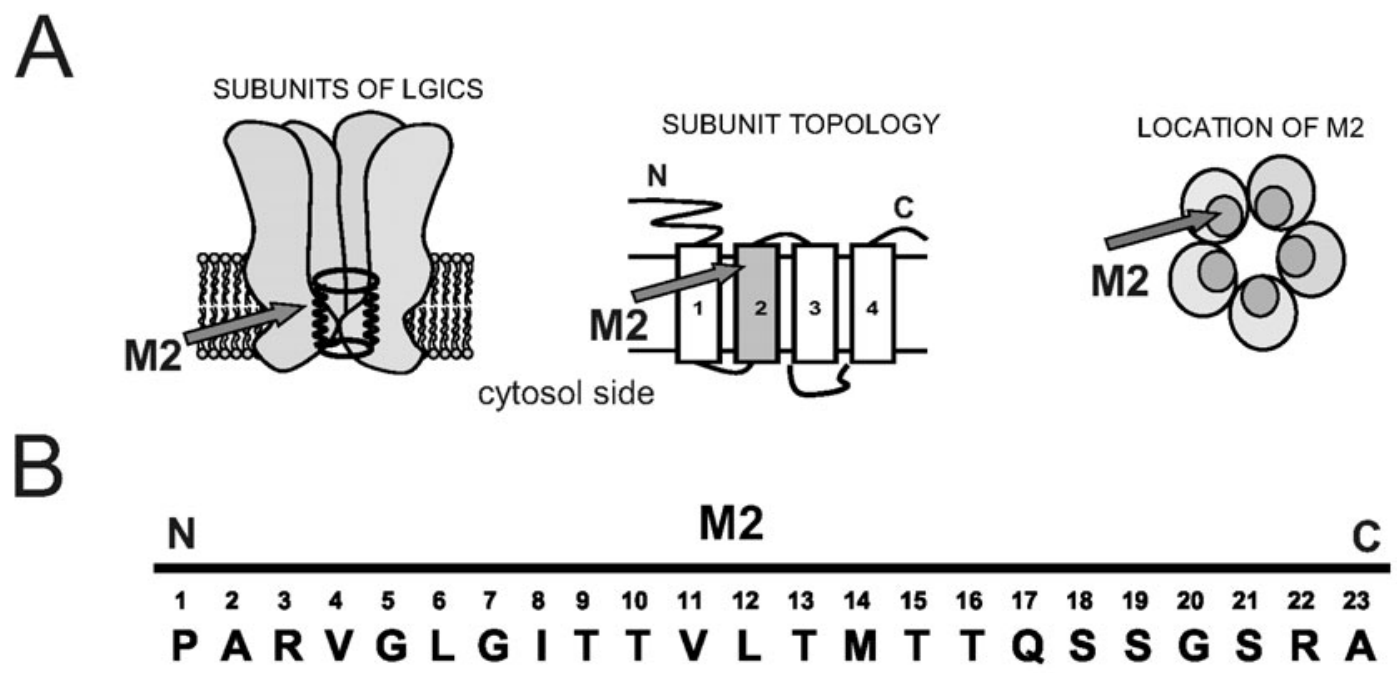

Figure 1. Structure of the ligand-gated ion channel superfamily and amino-acid sequence of M2 peptide.

(A) Five protein subunits of LGICS, the classic model showing transmembrane subunit topology of hydrophobic segments M1-M4 and schematic structure showing the location of M2 lining the pore. (B) Amino-acid sequence of the synthetic M2 peptide of the glycine receptor.

the ion-selectivity of the M2 pore. It has been shown that two arginine residues at the $\mathrm{N}$ and $\mathrm{C}$ termini of M2 (positions 3 and 22) contribute to the anion selectivity of the channel. M2 forms a cation selective pore when these arginine residues are substituted with glutamic acid (Reddy et al., 1993).

It has been shown that the membrane surface charge modifies the conductance of multi-state channels formed in planar lipid bilayers by the peptide antibiotic alamethicin (Aguilella \& Bezrukov, 2001). In our studies, we focused on the influence of the bilayer composition on M2 pore properties.

The synthetic M2 peptide was reconstituted into black lipid membranes and the current characteristic for an ion channel was ob- ductance states of the M2 pore. The reversal potential measured in the $150 / 600 \mathrm{mM} \mathrm{KCl}$ gradient is equal to $-19 \mathrm{mV}$ and this shift proves that the examined pore is anion-selective. The permeability ratio for $\mathrm{P}_{\mathrm{K}} / \mathrm{P}_{\mathrm{Cl}}$ is 0.25 . The current-time traces were also recorded in the $150 / 600 \mathrm{mM} \mathrm{KCl}$ gradient (cis/trans) at different voltages (Fig. 3A) for membranes containing phospholipids PE:PS = 1:3 $(n=10)$. Fig. 3B shows current-voltage curves for single channel openings at different voltages under these conditions. Solid and dashed lines indicate the main-conductance and sub-conductance states of the M2 pore. The reversal potential measured in the $150 / 600 \mathrm{mM} \mathrm{KCl}$ gradient is equal to $20 \mathrm{mV}$ and this shift proves that the 

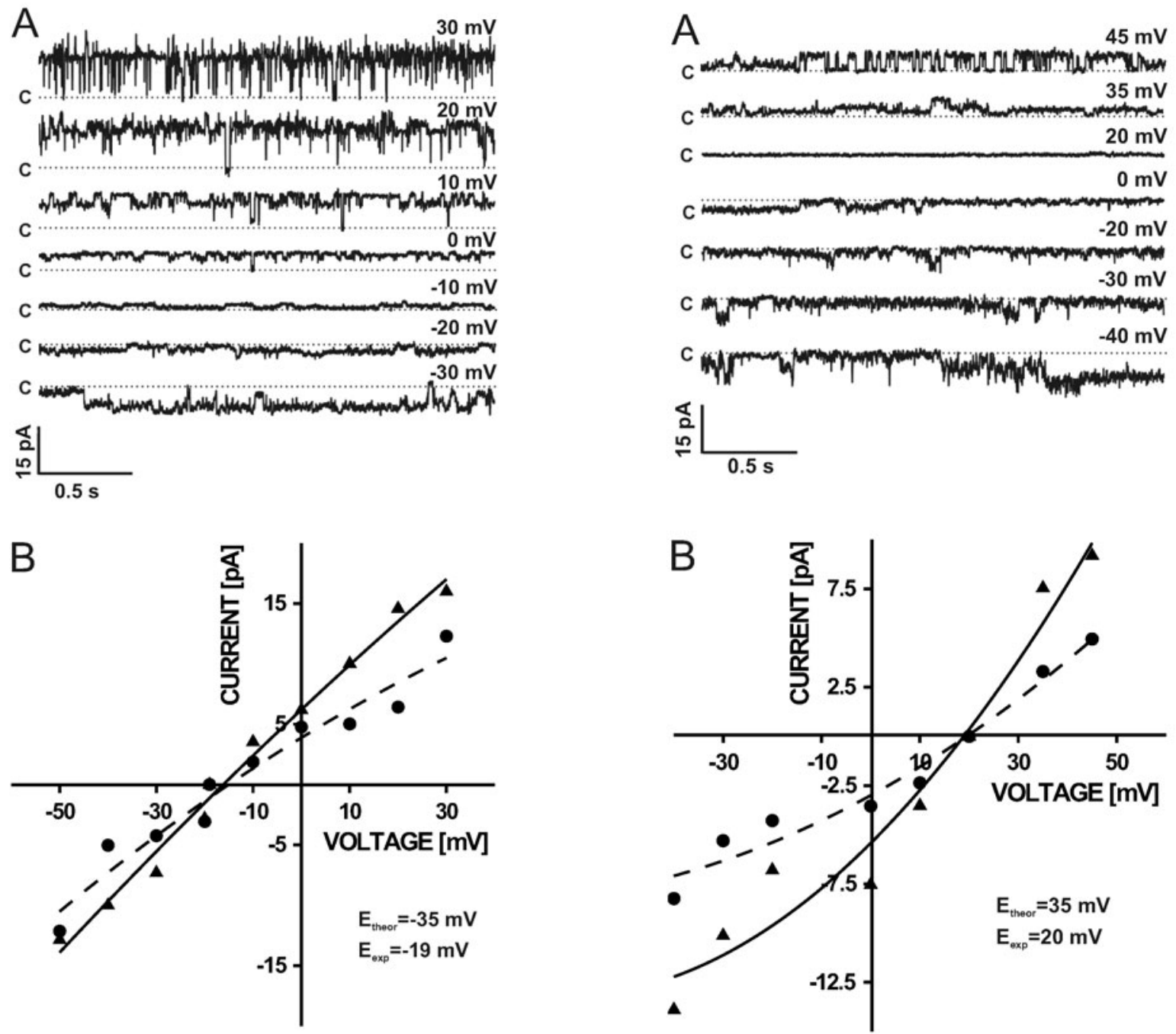

Figure 2. Single-channel recordings of segment M2 pore in $\mathbf{7 5} \% \mathrm{PE}$ and $\mathbf{2 5 \%} \mathrm{PS}$ planar lipid bilayers.

(A) Recordings were made in $150 / 600 \mathrm{mM} \mathrm{KCl}$ gradient (cis/trans), $20 \mathrm{mM}$ Hepes, $\mathrm{pH}$ 7.0, at different voltages $(n=5)$. C indicates the closed channel state. (B) Current-voltage characteristics of single-channel events in the M2 pore. Solid and dashed lines indicate substates of the M2 pore. Recordings were low pass filtered at $200 \mathrm{~Hz}$.

examined pore, in contrast to the results obtained from the data in Fig. 2, is cation-selective. The permeability ratio for $\mathrm{P}_{\mathrm{K}} / \mathrm{P}_{\mathrm{Cl}}$ is equal to 4.36 . The single-channel currents were recorded with two predominant amplitudes corresponding to the main-conductance and sub-conductance states. Both conductance states (about $12 \mathrm{pS}$ and $30 \mathrm{pS}$ ) were measured in a symmetric solution of 50 $\mathrm{mM} \mathrm{KCl}$. The planar lipid bilayer was com-

Figure 3. Single-channel recordings of the segment M2 pore in $25 \% \mathrm{PE}$ and $75 \%$ PS planar lipid bilayers.

(A) Recordings were made in $150 / 600 \mathrm{mM} \mathrm{KCl}$ gradient (cis/trans), $20 \mathrm{mM}$ Hepes, $\mathrm{pH}$ 7.0, at different voltages $(n=10)$. C indicates closed channel state. (B) Current-voltage characteristics of single-channel events of the M2 pore. Solid and dashed lines indicate substates of the M2 pore. Recordings were low pass filtered at $200 \mathrm{~Hz}$.

posed of $100 \%$ phosphatidylserine (not shown).

In summary, we used the M2 peptide of the glycine receptor as a model system for the pore-forming structure of an ion channel. We observed an ionic current and two predominant amplitudes corresponding to the main-conductance and sub-conductance states. The permeability for cations, calculated from the reversal potential measure- 
ments with concentration gradients of $\mathrm{KCl}$, is equal to 0.25 , indicating that the species carrying most of the current is $\mathrm{Cl}^{-}$. In contrast, in negatively charged planar membranes, the M2 pore is a cation-selective channel. The permeability for cations over anions is equal to 4.36. We have shown that the M2 segment incorporated into a lipid bilayer forms a pore with two conducting substates. It was also shown that the selectivity of the pore critically depends on lipid composition. The more net negatively charged lipid molecules are in the bilayer the lower anion and higher the cation concentration is near the membrane surface. The increased concentration of cations near the entrance to the pore increase the probability of cations flowing through the pore. Changing the lipid composition from PE:PS = 3:1 to PE:PS $=1: 3$ increases the permeability ratio of potassium cations over chloride anions 17.5-fold.

An electrophysiological study in which purified glycine receptor was incorporated into a planar bilayer consisting of a neutral phospholipid (Cascio et al., 2001) and studies of native glycine receptors by the patch-clamp technique (Bormann et al., 1987) showed a functional glycine-gated ion channel. The properties of the ion channel, it is preferential permeability to anions and sub-conductance levels were very similar to our observations.

In conclusion, the results provide evidence that the composition of the lipid bilayer is an important factor determining the ion selectivity of the M2 peptide. The electrostatic effect of charged lipid molecules on the pore formed by M2 seems to be similar to the effect of charged amino-acid residues on the ion selectivity of channels of the LGICS family, i.e. net negatively charged channels are cation specific and net positively charged channels are anion specific. In support of this hypothesis, the net charge of the cation specific nAChR-gated is (-69) elementary charges (Karlin, 1993), the anion specific GABA $_{A}$ R-gated is (+25) (Tyndale et al., 1995), and the anion specific GlyR-gated channel is (+60) (Langosch, 1995).

It was previously shown that anion channel blockers, 9-anthracene carboxylic acid (9-AC) and influmic acid (NFA), blocked the activity of the anion selective $\mathrm{T}_{4} \mathrm{M} 2$ pore but the anesthetic lidocaine (QX-222), a cation channel blocker (Reddy et al., 1993), did not. It will be important to establish whether 9-AC and NFA influence the channel activity of the M2 peptide under anion selective and cation selective conditions. Moreover, it was shown, using circular dichroism spectra, that the M2 peptide was able to change its structure in various mixtures of trifluoroethanol and phosphate buffer (Langosch et al., 1991). It is likely that changes in the secondary structure of the M2 peptide in lipid bilayers are able to influence the pore selectivity.

Ms Anna Kicinska is kindly acknowledged for help on the black lipid membrane technique.

\section{R E F E R E N C E S}

Adcock C, Smith GR, Sansom MS. (1998) Electrostatics and the ion selectivity of ligand-gated channels. Biophys J.; 75: 1211-22.

Aguilella VM, Bezrukov SM. (2001) Alamethicin channel conductance modified by lipid charge. Eur Biophys J.; 30: 233-41.

Anzai K, Takano C, Tanaka K, Kirino Y. (1994) Asymmetrical lipid charge changes the subconducting state of the potassium channel from sarcoplasmic reticulum. Biochem Biophys Res Commun.; 15: 1081-7.

Bormann J, Feigenspan A. (1995) GABAC receptors. Trends Neurosci.; 18: 515-9.

Bormann J, Hamill OP, Sakmann B. (1987) Mechanism of anion permeation through channels gated by glycine and gamma-aminobutyric acid in mouse cultured spinal neurones. J Physiol.; 385: 243-86. 
Cascio M, Shenkel S, Grodzicki RL, Sigworyh FJ, Fox RO. (2001) Functional reconstitution and characterization of recombinant human $\alpha_{1}$-glycine receptors. J Biol Chem.; 276: 20981-8.

De Godoy CMG, Cukierman S. (2001) Modulation of proton transfer in the water wire of dioxolane-linked gramicidin channels by lipid membranes. Biophys J.; 81: 1430-8.

Fan Z, Makielski JC. (1997) Anionic phospholipids activate ATP-sensitive potassium channels. J Biol Chem.; 272: 5388-95.

Hille B. (2001) Selective permeability: independence. In Ion channels of excitable membranes. pp 441-70. Sinauer Associates Inc., Sunderland, U S A.

Karlin A. (1993) Structure of nicotinic acetylcholine receptors. Curr Opin Neurobiol.; 3: 299-309.

Keramidas A, Moorhouse AJ, Pierce KD, Schofield PR, Barry PH. (2002) Cation-selective mutations in the M2 domain of the inhibitory glycine receptor channel reveal determinants of ion-charge selectivity. $J$ Gen Physiol.; 119: 393-410.

Kuhse J, Betz H, Kirsch J. (1995) The inhibitory glycine receptor: architecture, synaptic localization and molecular pathology of a postsynaptic ion-channel complex. Curr Opin Neurobiol.; 5: 318-23.

Langosch D. (1995) Inhibitory glycine receptors. In Receptors and channels. Ligand- and voltage-gated ion channels. pp 291-305. CRC Press, Boca Raton.

Langosch D, Hartung K, Grell E, Bamberg E, Betz H. (1991) Ion channel formation by synthetic transmembrane segments of the inhibitory glycine receptor - a model study. Biochim Biophys Acta.; 18: 36-44.

Leite JF, Cascio M. (2001) Structure of ligand-gated ion channels: critical assessment of biochemical data supports novel topology. Mol Cell Neurosci.; 17: 777-92.

Nalecz MJ, Zborowski J, Famulski KS, Wojtczak L. (1980) Effect of phospholipid composition on the surface potential of liposomes and the activity of enzymes incorporated. Eur $J$ Biochem.; 112: 75-80.

Nicholls JG, Martin AR, Wallace BG, Fuchs PA. (2001) Structure of ion channels. In From neuron to brain. pp 39-59. Sinauer Associates Inc., Massachusetts, U S A.

Reddy GL, Iwamoto T, Tomich JM, Montal M. (1993) Synthetic peptides and four-helix bundle proteins as model system for the pore-forming structure of channel proteins. $J$ Biol Chem.; 268: 14608-15.

Reeves DC, Lummis SC. (2002) The molecular basis of the structure and function of the 5-HT3 receptor: a model ligand-gated ion channel. Mol Membr Biol.; 19: 11-26.

Rostovtseva TK, Aguilella VM, Vodyanoy I, Bezrukov SM, Parsegian VA. (1998) Membrane surface-charge titration probed by gramicidin A channel conductance. Biophys J.; 75: 1783-92.

Sieghart W. (1995) Structure and pharmacology of gamma-aminobutyric acid A receptor subtypes. Pharmacol Rev.; 47: 181-234.

Sieghart W, Sperk G. (2002) Subunit composition, distribution and function of GABA(A) receptor subtypes. Curr Top Med Chem.; 2: 795-816.

Sprong H, van der Sluijs P, van Meer G. (2001) How proteins move lipids and lipids move proteins. Nat Rev Mol Cell Biol.; 2: 504-13.

Twyman RE, Macdonald RL. (1991) Kinetic properties of the glycine receptor main- and sub-conductance states of mouse spinal cord neurons in culture. J Physiol.; 435: 303-31.

Tyndale RF, Olsen RW, Tobin AJ. (1995) $\mathrm{GABA}_{\mathrm{A}}$ receptors. In Receptors and channels. Ligand-and voltage-gated ion channels. pp 265-90. CRC Press, Boca Raton.

Wallace DP, Tomich JM, Eppler JW, Iwamoto T, Grantham JJ, Sullivan LP. (2000) A synthetic channel-forming peptide includes $\mathrm{Cl}^{-}$secretion: modulation by $\mathrm{Ca}^{2+}$-dependent $\mathrm{K}^{+}$channels. Biochim Biophys Acta.; 1464: 69-82. 